\title{
On a new sensing strategy using a combination of ultrasonic and photoacoustic techniques
}

\author{
Jan Niemi* , Torbjörn Löfqvist*, and Per Gren ${ }^{\dagger}$ \\ ${ }^{*}$ Department of Computer Science and \\ Electrical Engineering \\ ${ }^{\dagger}$ Division of Experimental Mechanics \\ Lulea University of Technology, Sweden \\ *Email: jan.niemi@ltu.se
}

\begin{abstract}
In the process industry there is a need for development of new or improved in-line sensor techniques determining bulk properties of a suspension or different physical properties of the suspended particles. In this study we present a sensing strategy where both acoustical and photoacoustical measurement techniques are integrated and applied to aqueous fibre suspensions.

The two measurement techniques were tested experimentally in a custom made measurement cell using Nylon 66 fibres suspended in distilled and degassed water to mass fractions of $0.12 \%$ and $0.25 \%$. The result shows two different behaviors. The attenuation in the transducer generated pulse is proportional to mass fraction in the suspension. And the photoacoustical signal is sensitive to the amount of scatterers, i.e fibres, in the suspension. Further work is required to determine this relation. If the relation is established, the combination of these techniques have the potential to give the mass fraction as well as the particle number density in an aqueous fibre suspension.
\end{abstract}

\section{INTRODUCTION}

The process industry is today aiming for higher quality of their products. This raises the demand for more precise and advanced process control strategies. These strategies rely on quick and accurate sensing of various process variables. Considering processes where particles are suspended in a fluid, e.g. the paper and pulp, mineral, or biomedical industries, there is a need for development of new or improved in-line sensing techniques determining different physical properties of the particles or bulk properties of the suspension.

In the case of the pulp and paper industry the majority of measurement systems used today in characterisation of pulp fibre suspensions are based on either optical, microwave or mechanical measurement principles. These systems are basically used in measuring fibre mass fraction, geometrical properties of the fibres or some chemical properties of the pulp. In [1] it is shown that ultrasonic measurement techniques have a potential to complement the existing measurement principles by characterizing the mechanical properties of the suspended fibres in paper pulp. Furthermore, in a study by [2] it is shown that a combination of optical and ultrasonic techniques can be used in determining the mass fractions of fibres and fines respectively in a pulp sample. However, In [2] two different experimental setups and measurement cells were used. For the optical measurements a time-of-flight measurement of a short light pulse was used in conjunction with ultrasonic attenuation spectra from a transducer based pulse-echo technique. In this study we present a sensing strategy where both acoustical and photoacoustical measurement techniques are integrated. For this purpose have we designed and built a custom made measurement cell.

\section{Cell Design}

The measurement cell is designed to permit measurements of the acoustic waves generated from an ultrasonic transducer as well as the acoustical waves produced from a photoacoustic process when pulsed laser light is interacting with the suspension.

The cell is made of four precision machined rectangular pieces of stainless steel. Each piece is 50 by $100 \mathrm{~mm}$ and $10 \mathrm{~mm}$ thick. The pieces are assembled to form a rectangular box, $100 \mathrm{~mm}$ long and a quadratic sample space with a side length of $30 \mathrm{~mm}$. At each end of the cell, plates of brass are mounted. To the end plates a recirculating system can be connected. To reduce the problem of having microbubbles stick to the sides, all sides inside the cell are polished to a mirror quality. This also reduces the photoacoustic noise. The

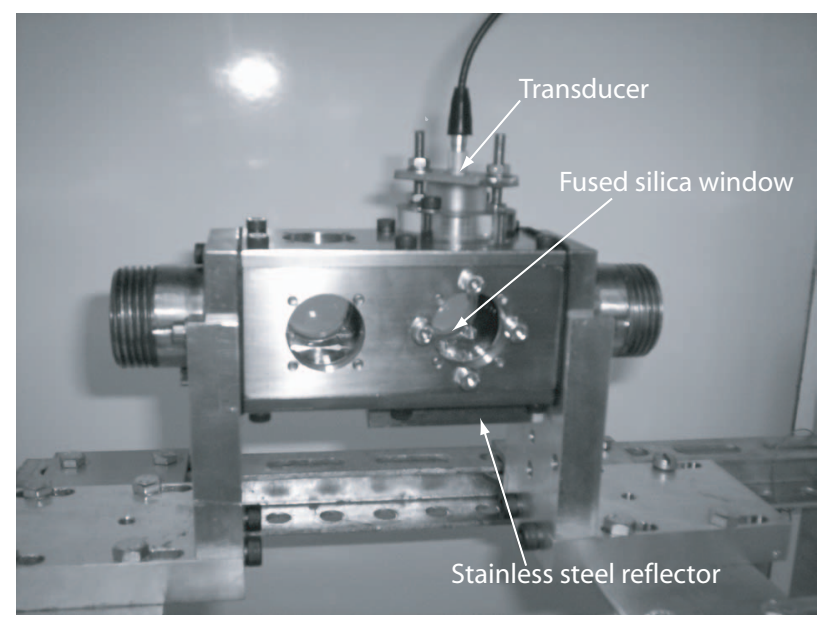

Fig. 1. The custom made measurement cell used in this study

cell is designed for two different modes of measurement. The first, the optical mode, consists of four optical windows made of fused silica with the diameter of $25 \mathrm{~mm}$ placed on each 
side of the cell. This option can be used to determine the optical properties of the suspension, for instance using time-offlight measurements to establish the scattering properties of the suspension. However, the possibilities the optics option gives is not explored in the present study. The second mode, the acoustical mode, consists of an ultrasonic transducer fitted to a holder that is attached to one side of the cell. On the opposite side there is a stainless steel reflector. The outer side of the stainless steel reflector is at oblique angle in order to prevent any acoustic echoes to interfere with the acoustic echoes inside the cell. Placed along an orthogonal axis to the transducer set-up are two optical windows where the pulsed laser light enters and exits the cell. The laser light creates, through a rapid heating and cooling process, the acoustic signal that the ultrasonic transducer receives.

\section{A. Experimental setup}

In the transducer based mode, the well known pulse-echo technique is used. A pulser/receiver 5073PR manufactured by Panametrics, Waltham, MA, USA, is set to excite the transducer and amplify the received signal. The transducer is a broadband PVDF transducer with a centre frequency of $25 \mathrm{MHz}$, model IA-FM25.3, manufactured by GE Inspection Technologies, USA. The pulse travels through the suspension and reflects back at the stainless steel reflector. Using this option the laser is inactive.

In the photoacoustic mode the pulser/receiver is set in receive mode and is trigged by the laser trigger unit. The light is generated from a twin-cavity, injection-seeded, pulsed Nd:YAG laser, Spectron SL804T from Spectron Laser Systems, Rugby, United Kingdom. The wavelength is $532 \mathrm{~nm}$. The pulse duration is $10 \mathrm{~ns}$. The pulse energy was estimated to $10 \mathrm{~mJ} / \mathrm{pulse}$ at a repetition rate of $10 \mathrm{~Hz}$. The light was focused using a plano-convex lens having a focal length of $\mathrm{f}=50 \mathrm{~mm}$. The laser beam enters the cell through the fused silica windows, model 02WLQ105 manufactured by Melles Griot. The laser beam is crossing the cell orthogonally to the acoustic axis of the ultrasonic transducer set-up. The ultrasonic transducer is receiving the acoustical signals, or echoes, which are generated when the photons are absorbed by the suspension under investigation. The first echo is the acoustic signal which directly enters the transducer. The second echo is the acoustic signal that has been reflected at the steel reflector before reaching the transducer, see figure 2 . In both cases the received signal is digitized using a CompuScope 12400 oscilloscope card, by Gage Applied Technologies Inc., Lachine, QC, Canada, with 12-bit resolution and a sampling rate of $400 \mathrm{MHz}$. All data was stored in a computer for off-line analysis. The resulting timedomain waveforms were calculated off-line using the average of 100 sampled waveforms to reduce random noise. Before the averaging process, the sampled waveforms are aligned to reduce timing jitter by employing a method proposed by [3]. A digital thermometer S2541, by Systemteknik AB, Sweden, monitored the temperature both in the suspension under test and in the room. The temperature in the suspensions under test was $21.7 \pm 0.2{ }^{\circ} \mathrm{C}$.

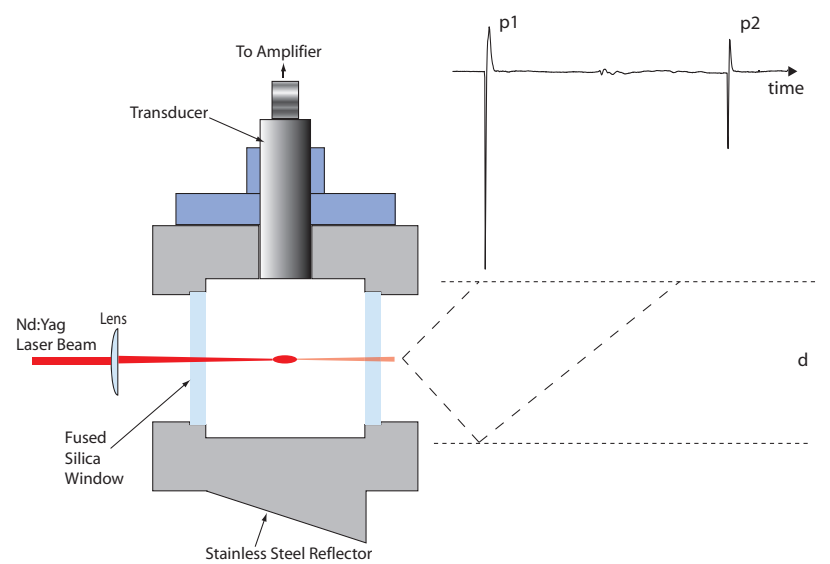

Fig. 2. Cross section of the measurement cell at the part of the cell used in this study. Here the photoacoustic mode is viewed

To determine the distance between the transducer and the reflector, pure, distilled and degassed water was used as a reference since it has a well known relationship between speed of sound and temperature [4]. Using the temperature of the reference liquid and a cross-correlation technique to determine the time-of-flight for the transducer generated pulse that has travelled back and forth once respectively twice in the cell. The distance was found to be $0.03035 \pm 0.00005 \mathrm{~m}$.

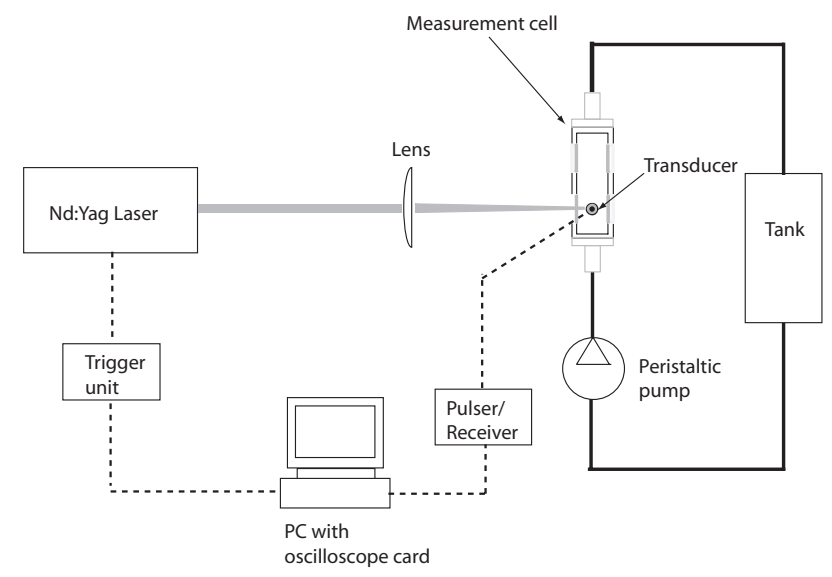

Fig. 3. Experimental setup for the photoacoustic method. In the transducer based method the laser is switched off

The suspension sample was pumped in a recirculating system shown in figure 3 . The pump was a peristaltic pump model 401-120-012-130-SB manufactured by Williamson Pumps Ltd, United Kingdom. To reduce the temperature rise caused by the pumping the suspension was only pumped just before and during the time of measurement.

Both techniques, the transducer based and the laser based photoacoustic were tested experimentally in the cell using aqueous suspensions of Nylon 66 fibres, manufactured by Swissfloc, Emmenbrücke, Switzerland to mass fractions of $0.12 \%$ and $0.25 \%$. The fibres are dtex 1.7 with a diameter of $17 \pm 0.54 \mu \mathrm{m}$ and a length of $1.2 \mathrm{~mm}$, dtex 17 with a diameter of $51.07 \pm 0.64 \mu \mathrm{m}$ and a length of $1.5 \mathrm{~mm}$ and dtex22 with a 
diameter of $55.17 \pm 0.91 \mu \mathrm{m}$ and a length of $1.5 \mathrm{~mm}$.

\section{B. Results}

In figure 4 the spectrum of the received acoustical signal from pure water of each method is shown. The frequency content in the photoacoustic pulse is significantly lower than the corresponding transducer excited pulse. In our case the energy in the laser pulse created a dielectric breakdown, which is believed to result in a comparatively low frequency content in the photoacoustic pulse.

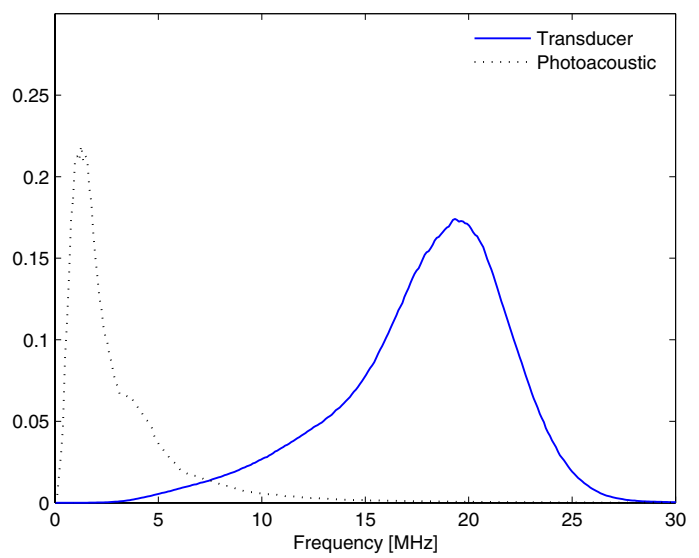

Fig. 4. The frequency spectra of the transducer generated and the photoacoustic pulses in water. The solid line is the transducer based method and the dashed is is the photoacoustic method

An important property to determine is the acoustic attenuation spectra. The attenuation in the sample was obtained by using pure water as reference. This gives,

$$
\alpha_{s}(\omega)=\frac{1}{2 d} \ln \left[\frac{\left|P_{w}(\omega)\right|}{\left|P_{s}(\omega)\right|}\right]+\alpha_{w}(\omega)
$$

where $\alpha_{s}(\omega)$ is the attenuation in the suspension, $d$ is the distance travelled by the pulse, $P_{w}(\omega)$ is the amplitude in the frequency domain of the water reference echo, $P_{s}(\omega)$ is the amplitude in the frequency domain of the sample echo and $\alpha_{w}(\omega)$ is the attenuation in water and is assumed to be $25 \cdot 10^{-15} f^{2}$ [5], where $f$ is the frequency.

In figure 5 the attenuation spectra for the transducer based method is shown. The result shows a direct relation between attenuation and mass fraction. The existence of a linear relationship between attenuation and mass fraction for Nylon 66 fibres can not be verified here because of the few number of different mass fractions. However, others e.g. [6] have shown that if the suspended particles act independently the attenuation is directly proportional to the volume/mass fraction. This assumption is assumed to be valid here.

It is noted that distinct extremas, peaks, appear in the attenuation spectra for dtex 17 and dtex 22 samples. These local extremas are believed to originate from resonance in the fibres. It can easily be shown that the resonance peaks are not due to longitudinal waves along the fibre. The attenuation extremas

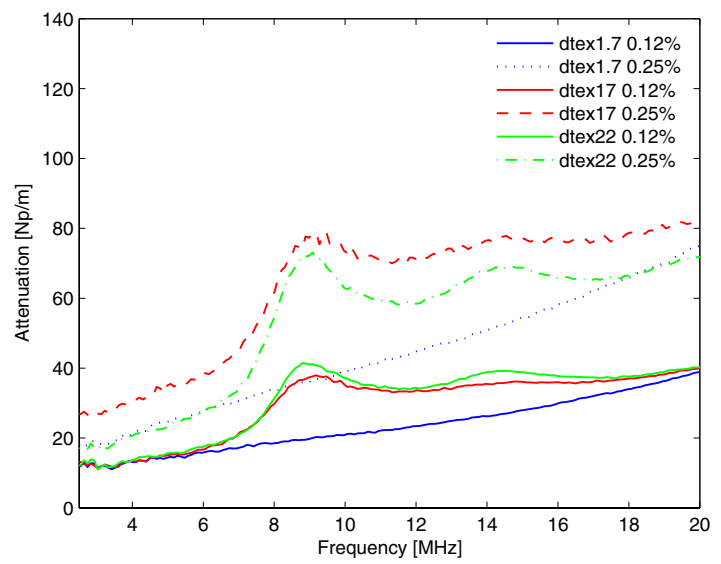

Fig. 5. The attenuation for the different samples using the transducer based method

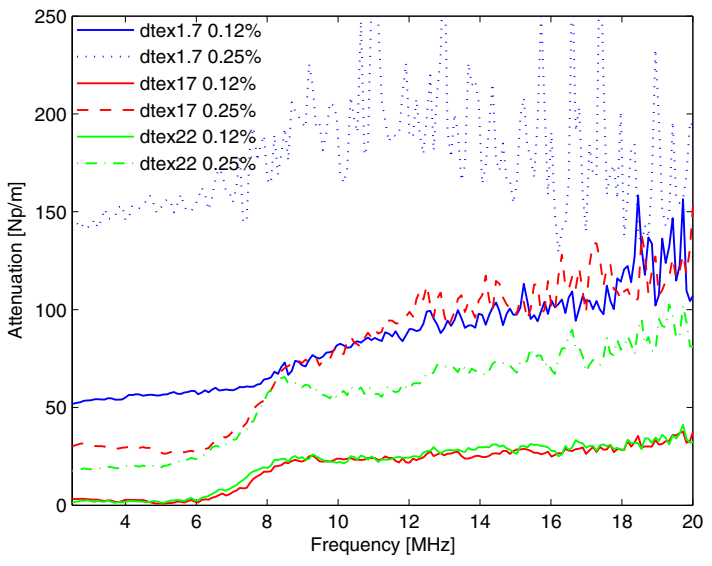

Fig. 6. The attenuation for the different samples using the photoacoustic method

could originate from radial oscillations of the fibre, which has been discussed in [7]. It is also noted that the attenuation spectra from the dtex $17,0.25 \%$ is significantly higher than the others. This is believed to originate from an unexpected variation in mass fraction due to flocculation of the suspension.

In the photoacoustic method the attenuation for the second echo is,

$$
\alpha_{\text {spa }}(\omega)=\frac{1}{d+d_{2}} \ln \left[\frac{\left|P_{w p a}(\omega)\right|}{\left|P_{\text {spa }}(\omega)\right|}\right]+\alpha_{w}(\omega)
$$

where $\alpha_{\text {spa }}(\omega)$ is the attenuation in the suspension, $d_{2}$ is the distance between the photoacoustical source and the steel reflector, $d$ is the distance between the steel reflector and the transducer, $P_{w p a}(\omega)$ is the amplitude in the frequency domain of the second water reference echo, $P_{\text {spa }}(\omega)$ is the amplitude in the frequency domain of the second sample echo and $\alpha_{w}(\omega)$ is the attenuation in water and is assumed to be $25 \cdot 10^{-15} f^{2}$, where $f$ is the frequency. In figure 6 the attenuation spectra for the photoacoustic method is shown. It shows similarities with the transducer based method for the location of the attenuation 
peaks. But the level of attenuation for the same sample differ from the transducer based method. Especially for the samples dtex $1.7,0.12 \%$ and $d t e x 1.7,0.25 \%$. This because the attenuation here is a combination of two different processes. The first is when the laser pulse scatters in the suspension and therefore reduces its energy which generates the photoacoustic pulse. The other process is the intrinsic acoustical attenuation. This result shows that the photoacoustic method is sensitive to the amount of scatters i.e. number of fibres in the suspension. In figure 7 the received signal of the second echo is shown. It shows that the amplitude of the signal has an inverse relation to the number of fibres in the suspension. Assuming that the relationship is linear the relation can be expressed as $N_{d}=\frac{K}{A}$, where $N_{d}$ is the particle number density, $A$ is the measured peak amplitude and $K$ is a proportionality factor.

In table I the values from each of the measurements are presented. In table I the proportionality factor for the inversele relationship between the particle number density and the amplitude of the received signal is calculated. If there were a linear correlation between the number of particles and the amplitude the proportionality factor should be constant. This is indicated by the results. However, one of the samples differ considerably $d$ te $x 1.7,0.12 \%$. As mentioned above, the reason for this is believed to originate from variations in mass fraction due to flocculation.
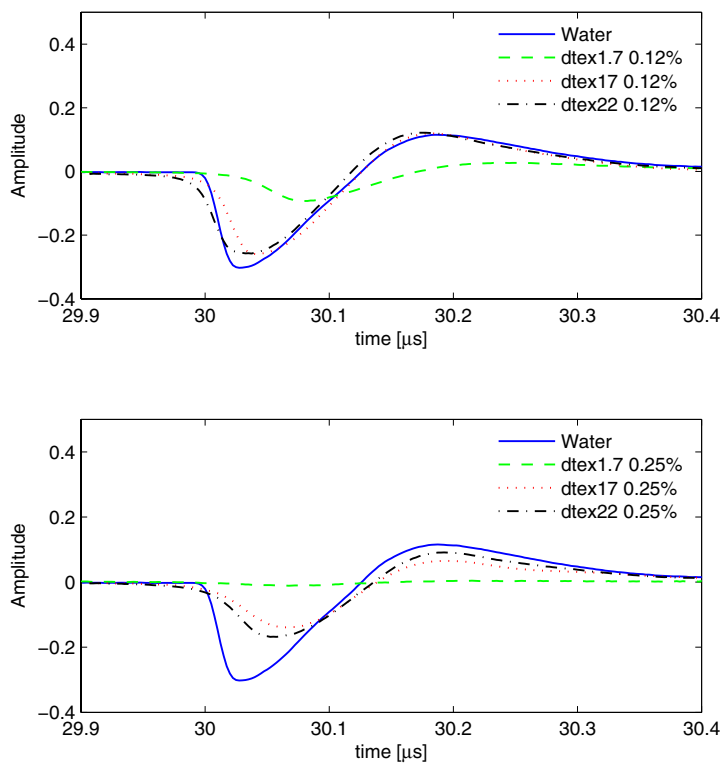

Fig. 7. Photoacoustic signals of the second echo that has been travelling through the suspension and reflected at the steel reflector and travelling back to the transducer.The upper figure shows the samples with mass fraction of $0.12 \%$ and the lower figure the samples with mass fraction of $0.25 \%$

\section{CONCLUSION}

In this study we have presented a new custom built measurement cell. The cell permits the simultaneous use of two
TABLE I

TABLE OF THE RESULT

\begin{tabular}{c|c|l|c|c}
\hline Fibre & Mass fraction & $\begin{array}{l}\text { Particle } \\
\text { Number } \\
\text { Density } \\
n / \mathrm{cm}^{3}\end{array}$ & Amplitude & K \\
\hline dtex1.7 & 0.12 & 3723 & 0.093 & $V \times n / \mathrm{cm}^{3}$ \\
dtex1.7 & 0.25 & 7756 & 0.011 & 346 \\
dtex17 & 0.12 & 342 & 0.260 & 85 \\
dtex17 & 0.25 & 714 & 0.139 & 99 \\
dtex22 & 0.12 & 294 & 0.258 & 76 \\
dtex22 & 0.25 & 612 & 0.168 & 103 \\
\hline
\end{tabular}

different measurement techniques, optical and acoustical. In the present study we use the ultrasonic signal generated from an ultrasonic transducer as well as the ultrasonic, or photoacoustic, signal generated using a pulsed laser. The two measurement techniques were tested experimentally in the cell using aqueous suspensions of Nylon 66 fibres suspended in distilled and degassed water to mass fractions of $0.12 \%$ and $0.25 \%$.

The result indicate that combination of these two techniques can be used in resolving the proportion of particles in a suspension. The transducer generated pulse can also be used to measure mass fraction in a suspension. For the particle measurement the result shows that the photoacoustic pulse is sensitive to the amount of scatters i.e fibres in the suspension. If the relation is established, the combination of these techniques have the potential to determine the mass fraction and the particle number density simultaneously.

\section{FURTHER WORK}

Since only a few samples were used in this study an extension is required where different mass fraction and fibre sizes are used. It is also important to ascertain if a linear relationship between the attenuation and mass fraction of suspended Nylon 66 fibres exists.

\section{REFERENCES}

[1] T. Lofqvist, "Ultrasonic wave attenuation and phase velocity in a paperfibre suspension," in Proc.IEEE Ultrasonics Symposium, vol. 1, 1997, pp. 841-844.

[2] M. Törmänen, J. Niemi, T. Löfqvist, and R. Myllylä, "Pulp consistency determined by a combination of optical and acoustical measurement techniques," Measurement Science and Technology, vol. 17, no. 4, pp. 695-702, 2006.

[3] A. Grennberg and M. Sandell, "Estimation of subsample time delay differences in narrowband ultrasonic echoes using the hilbert transform correlation," IEEE Transactions on Ultrasonics, Ferroelectrics, and Frequency Control, vol. 41, no. 5, pp. 588-595, 1994.

[4] N. Bilaniuk and G. S. K. Wong, "Speed of sound in pure water as a function of temperature," The Journal of the Acoustical Society of America, vol. 93, no. 4, pp. 2306-2306, 1993.

[5] L. Kinsler, A. Frey, A. Coppens, and J. Sanders, Fundamentals of Acoustics, 4th ed. John Wiley and Sons Inc., 2000.

[6] M. Stautberg Greenwood, J. Mai, and M. Good, "Attenuation measurements of ultrasound in a kaolin-water slurry: a linear dependence upon frequency," J. Acoust. Soc. Am. (USA), vol. 94, no. 2, pp. 908 - 16, 1993.

[7] Y. Aitomäki, "Towards a measurement of paper pulp quality : ultrasonic spectroscopy of fibre suspensions," Licenciate thesis, Luleå University of Technology, 2006. 